\title{
Aplicación del Mechanical Desktop en el Análisis dEEsfuerzos de Una Viga Empotrada
}

\author{
Recepción: Noviembre de 2004 / Aceptación: Diciembre 2004
}

\author{
(1) Luis Martínez Silva \\ (2) Oswaldo Rojas Lazo \\ (3) Luis Sampén Alquízar
}

\section{RESUMEN}

El presente artículo muestra una aplicación del análisis de esfuerzos usando el software Mechanical Desktop 6.0, a fin de determinar los esfuerzos en una viga sometida a una fuerza puntual. El desarrollo de los cálculos se establece aplicando una fuerza de $200 \mathrm{~N}$ a una viga de dimensiones $400 \times 300 \times 3000 \mathrm{~mm}$.

Palabras Claves: Ingeniería asistida por computadora (CAE). Esfuerzos en vigas. Aplicaciones en Mechanical Desktop 6.0.

The Application of Mechanical Desktop in an Embedded Beam Efforts Analysis

ABSTRACT

The present article shows an application of the efforts analysis using the Mechanical Desktop 6.0 Software, with the purpose of determining the efforts of a beam subjected to a punctual force. Calculations development is set by applying a $200 \mathrm{~N}$ force to a $400 \times 300 \times 3000 \mathrm{~mm}$ dimension beam.

Key words: Computer assisted engineering (CAE). Efforts in beams. Applications in Mechanical Desktop 6.0.

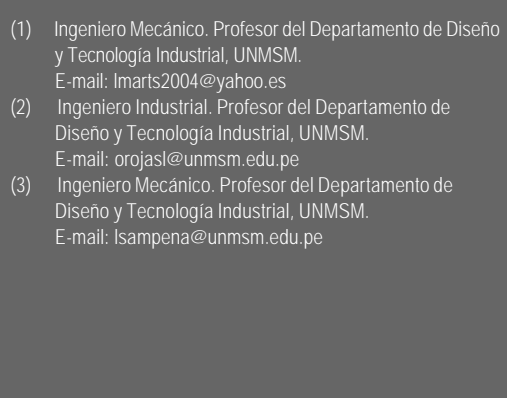

\section{INTRODUCCIÓN}

El desarrollo de la Ingeniería Asistida por Computadora (CAE) tiene su herramienta fundamental en el método por elementos finitos (FEA); El cual es un método numérico usado para resolver en forma aproximada ciertos problemas en ciencias e ingeniería.

Explicándolo en forma sencilla consiste en convertir un sólido en un numero finito de partes llamadas elementos, cuyo comportamiento se especifica con un numero finito de parámetros. Dichos elementos contienen una serie de puntos interconectados entre si llamados nodos y al conjunto se le conoce como malla (formada por triángulos).

\section{PASOS DE UN ANÁLISIS CON ELEMENTOS FINITOS}

Un análisis usando elementos finitos requiere de la disponibilidad de un programa de computación especializado en estos análisis. Existen numerosos programas de elementos finitos dentro de los cuales se pueden citar: PATRAN, NASTRAN, ABAQUS, COSMOS, ADINA, ANSYS, SAP, etc. que pueden resolver un gran rango de problemas en Ingeniería.

El uso de cualquiera de ellos para el análisis de un problema real sigue los siguientes pasos:

- Pre-procesamiento.

- Análisis propiamente dicho.

- Post-procesamiento.

- Interpretación de los resultados.

\section{APLICACIONES DEL MÉTODO DE LOS ELEMENTOS FINITOS}

Tiene aplicaciones casi ilimitadas, como ejemplo:

- Ingeniería y mecánica estructural, análisis sísmico.

- Mecánica del suelo, cimentaciones, mecánica de rocas.

- Hidrodinámica, ingeniería hidráulica, dinámica de fluidos.

- Ingeniería eléctrica.

- Ingeniería aeroespacial.

- Termodinámica.

- Ingeniería nuclear. 
- Diseño, análisis y prueba de prototipos.

- Medicina, etc.

A continuación se presenta una aplicación sencilla de una viga empotrada con carga puntual y posteriormente ver los resultados. Para esto se utiliza el software Mechanical Desktop Power Pack, software conocido y de amplia divulgación en el Perú.

\section{PROBLEMA}

Se trata de determinar los esfuerzos a los que se encuentra sometida la viga producto de la aplicación de una fuerza de 2000 Newton, inclinado a $45^{\circ}$. Tal como se muestra en la Figura 1.

Las dimensiones de la viga son: ancho $=300 \mathrm{~mm}$; altura $=400 \mathrm{~mm}$ y longitud de $3000 \mathrm{~mm}$. La viga de material acero clase $20 ; E=79979,1 \mathrm{~N} / \mathrm{mm}^{2} ; \mu$ (coeficiente de rugosidad $)=0,26 ; \rho($ densidad $)=7,72$ $\mathrm{Kg} / \mathrm{dm}^{3}$.

\section{Primer Paso}

Se desarrollará el modelo en Mechanical Desktop, es decir se construye la viga según las dimensiones dadas. Para esto, se debe cargar el Mechanical Desktop Power Pack. Y trabajar en el sistema métrico, el modelo se visualiza en la Figura 2.

\section{Segundo Paso}

Enseguida se da forma al problema, tal como muestra la Figura 3, indicando las fuerzas que actúan en la estructura y sus respectivos apoyos. Esto se conoce como el pre-procesamiento.

Para esto se hace clic en «Calculations» de la barra de herramientas «Content 3D». También, se debe tener activado la barra de herramientas «Content 3D». O también, del menú principal «Content 3D», opción: «Calculations» y luego la opción «FEA».
En la zona de texto se pide: «Select 3D-body», se selecciona la viga en cualquiera de sus puntos y luego se da «Enter».

También se selecciona el material haciendo clicken la opción: «Table», de la pantalla anterior, apareciendo otra pantalla, en donde se selecciona el material requerido (ver Figura 4).

Luego, se selecciona el material por defecto. Y se hace click en «Ok». Seguidamente, se debe ubicar los soportes de la viga, esto se hace en la pantalla anterior se selecciona del grupo del «Loads and Suports» la opción: $\triangle$ Después, Mechanical requiere de la cara del sólido y punto donde se dibujará los soportes (ver Figura 5).

Posteriormente, se puede ubicar el punto de aplicación de la fuerza y el valor de ésta en 2000 Newton. Para ver la solución se hace click en «Run Calculations" y Mechanical activará los iconos de «Results», en donde se puede elegir la forma de presentación de los resultados. Esto es el postprocesamiento. Se elige la opción, en este caso, se ingresa una fuerza de 2000 Newton a un ángulo de $45^{\circ}$ respecto a X. Tal como muestra la Figura 6 .

Para encontrar la Solución FEA, de la estructura se hace clicken: «Run Claculation». Esto vendría a ser el Análisis.

En la Figura 7 se puede observar 03 gráficos acompañados de una barra de colores:

- En el gráfico 1 se presenta la solución en colores, los colores representan los esfuerzos Von Mises.

- En el gráfico 2 se presenta La malla que se utiliza para el análisis.

- En el gráfico 3 se presenta el modelo a procesar.

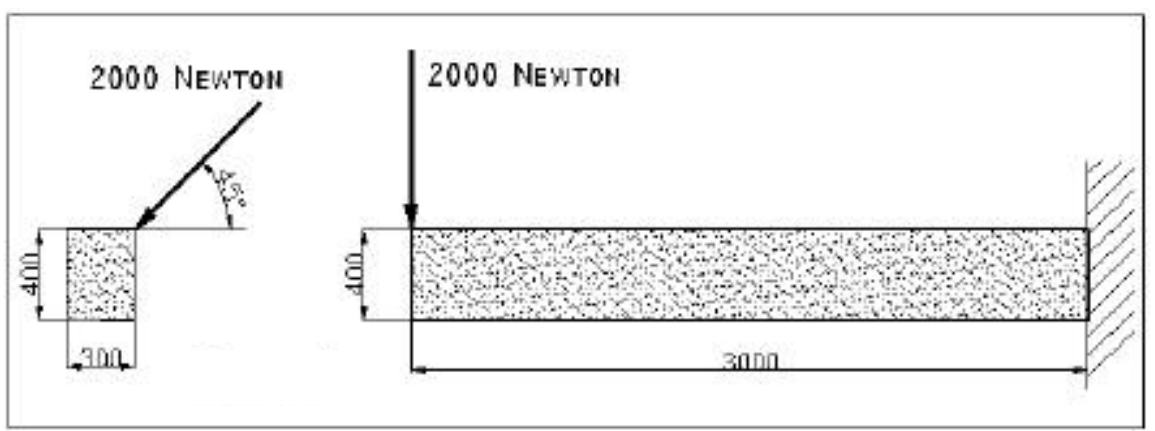

Figura 1. Vista de la viga sometida a una fuerza 


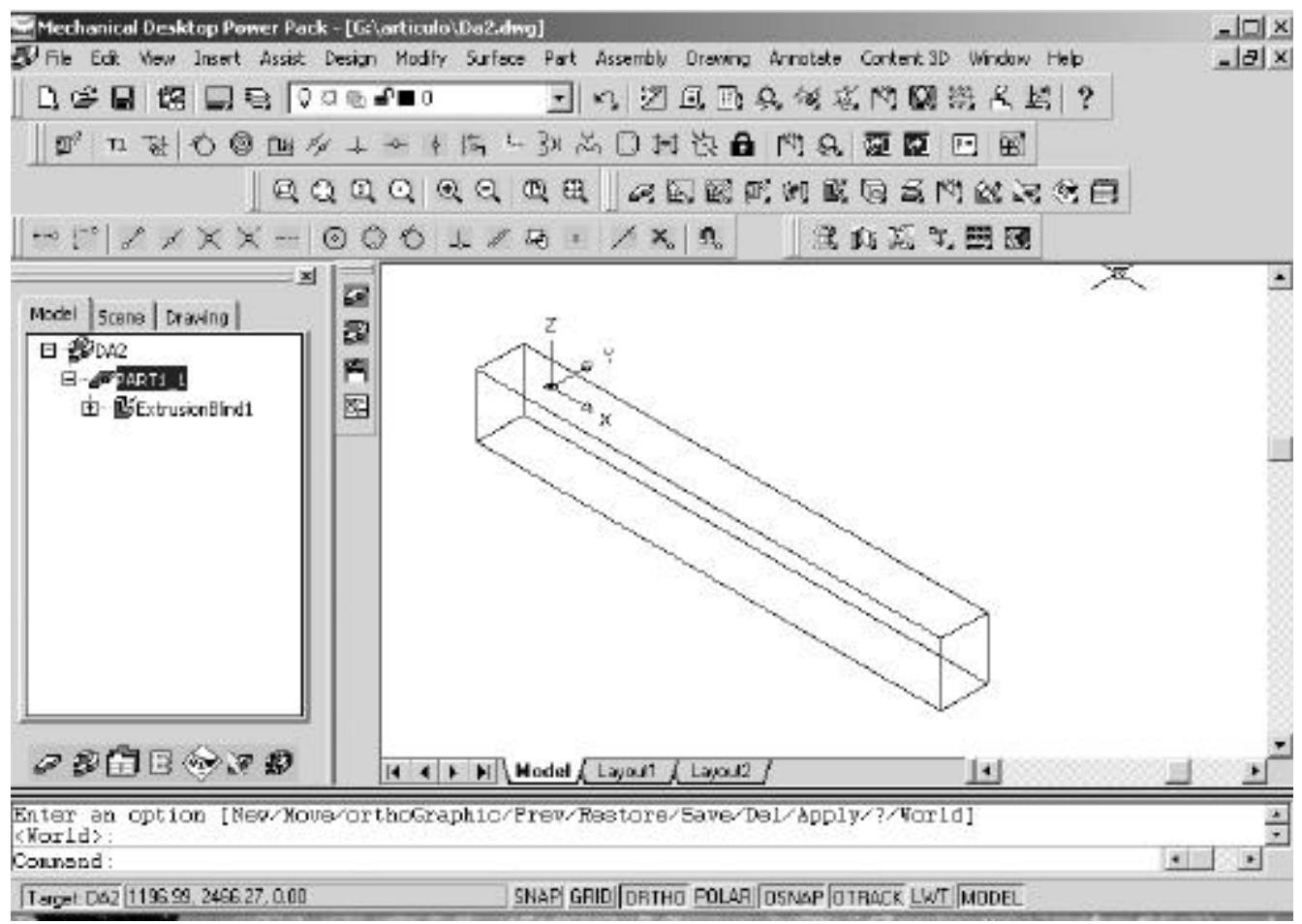

Figura 2. Configuración del software

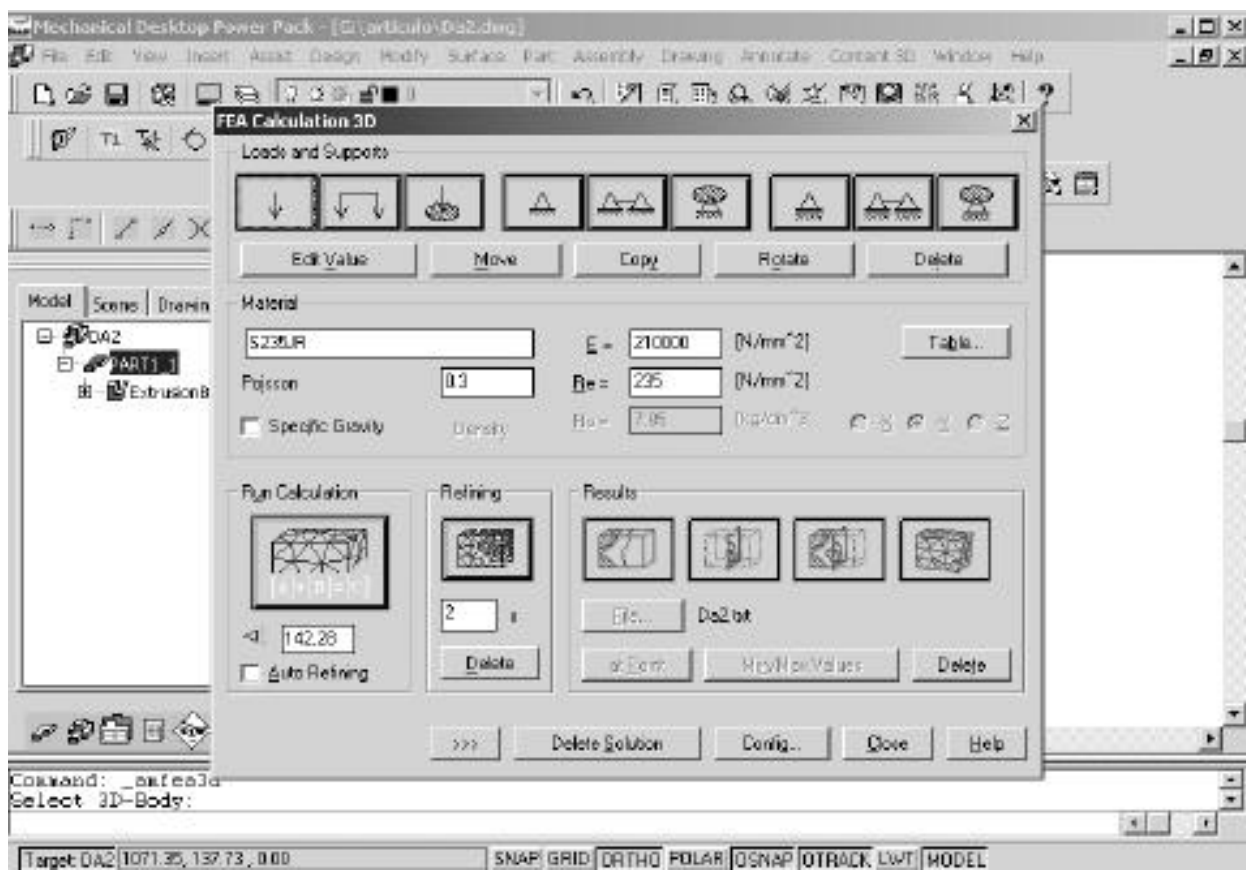

Figura 2. Pre-procesamiento 


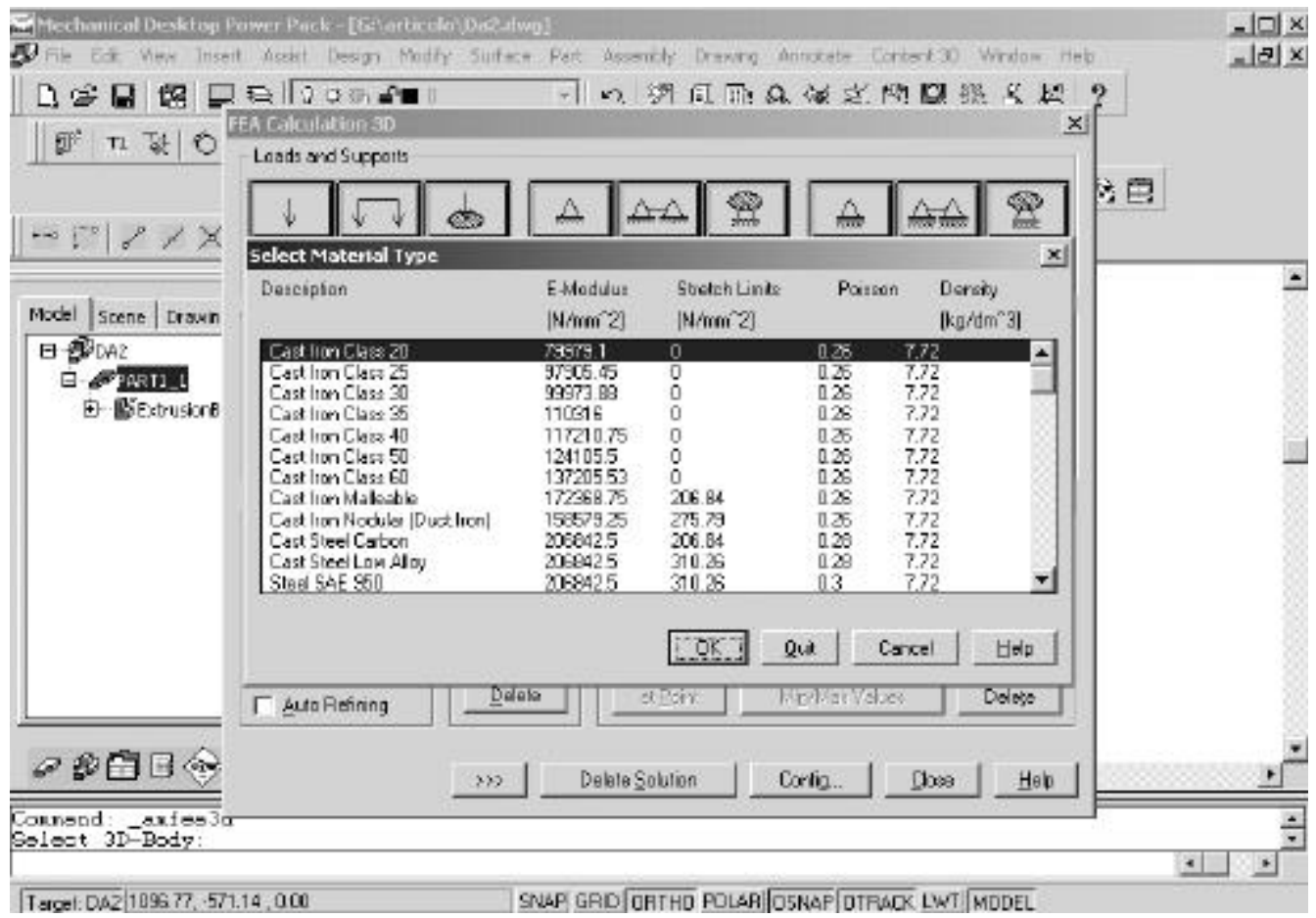

Figura 4. Selección del material requerido

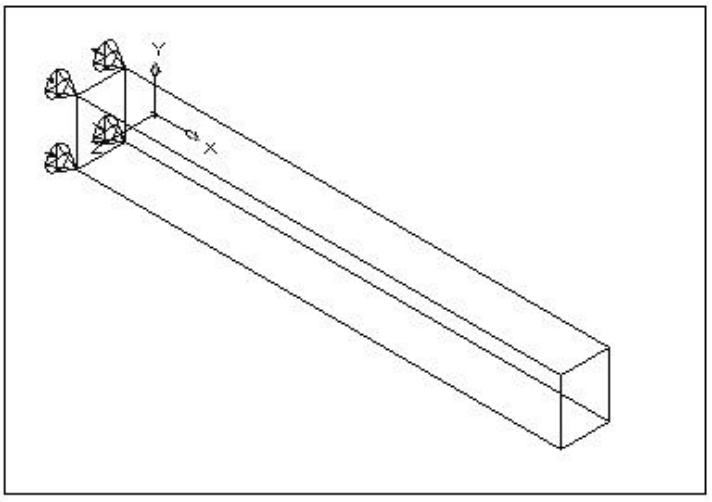

Figura 5. Requerimiento de la cara del sólido

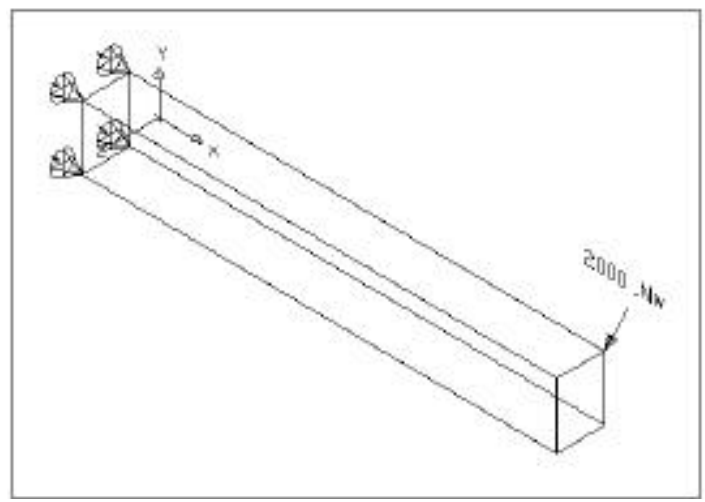

Figura 6. Ubicación del punto de aplicación de la fuerza 


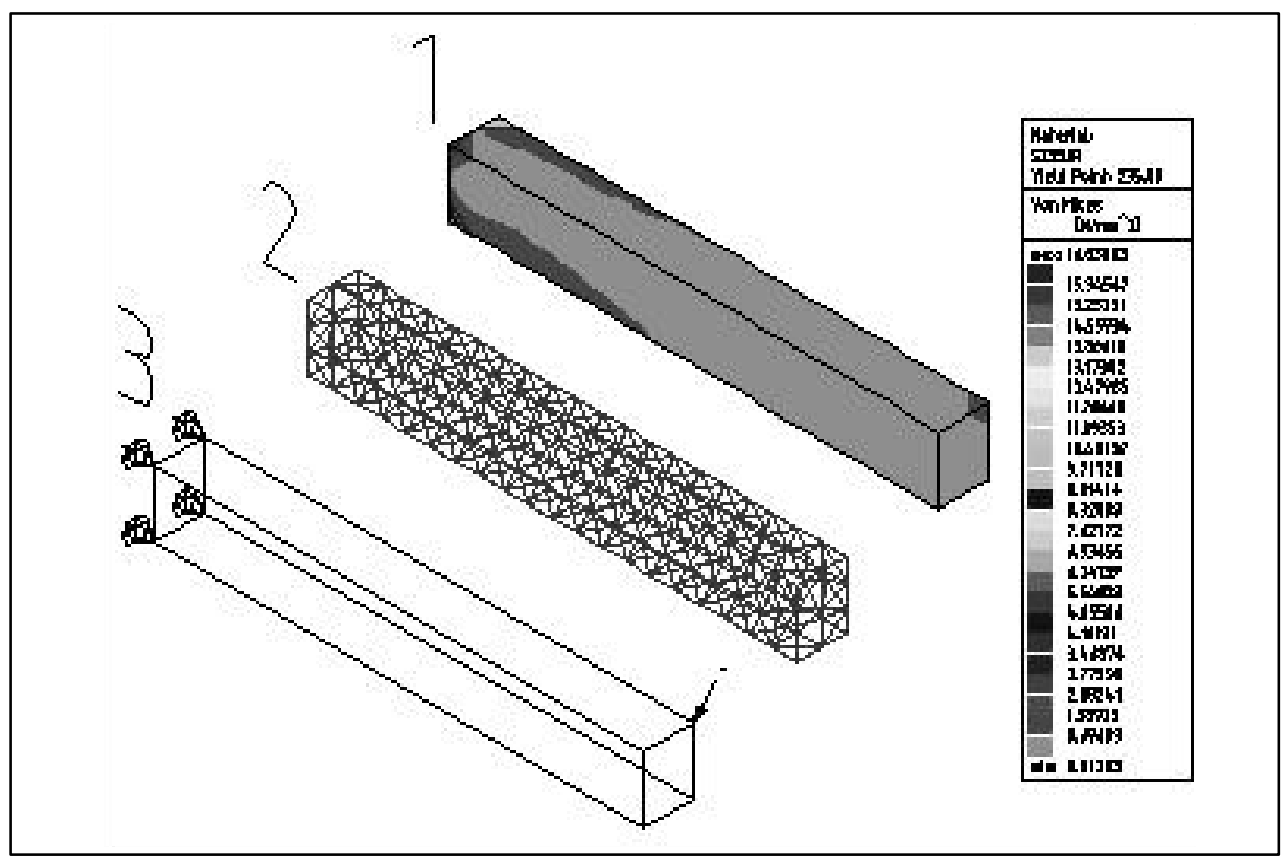

Figura 7. Selección del material requerido

\begin{tabular}{|c|c|c|c|c|}
\hline \multicolumn{5}{|c|}{ 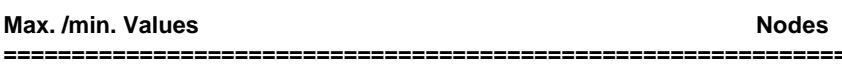 } \\
\hline Displacement & $\operatorname{Max} d x$ & [ $[\mathrm{mm}]:$ & 0.0135917 & 3 \\
\hline Displacement & $\operatorname{Max} d y$ & {$[\mathrm{~mm}]:$} & 0.0012417 & 272 \\
\hline Displacement & $\operatorname{Max} d z$ & {$[\mathrm{~mm}]$} & 0.0008291 & 268 \\
\hline Displacement & Max d & {$[\mathrm{mm}]:$} & 0.1351052 & 3 \\
\hline Tresca Stress & $\begin{array}{l}\text { Max sTr } \\
\text { Min sTr }\end{array}$ & $\begin{array}{l}{\left[\mathrm{N} / \mathrm{mm}^{\wedge} 2\right]:} \\
{\left[\mathrm{N} / \mathrm{mm}^{\wedge} 2\right]:}\end{array}$ & $\begin{array}{r}19.1903 \\
0.0034\end{array}$ & $\begin{array}{l}258 \\
0\end{array}$ \\
\hline Von Mises & $\begin{array}{l}\text { Max svM } \\
\text { Min svM }\end{array}$ & $\begin{array}{l}{\left[\mathrm{N} / \mathrm{mm}^{\wedge} 2\right]:} \\
{\left[\mathrm{N} / \mathrm{mm}^{\wedge} 2\right]:}\end{array}$ & $\begin{array}{r}16.6384 \\
0.0029\end{array}$ & $\begin{array}{l}258 \\
0\end{array}$ \\
\hline in direct. & $\begin{array}{l}\text { Max sx } \\
\text { Min sx }\end{array}$ & $\begin{array}{l}{\left[\mathrm{N} / \mathrm{mm}^{\wedge} 2\right]:} \\
{\left[\mathrm{N} / \mathrm{mm}^{\wedge} 2\right]:}\end{array}$ & $\begin{array}{l}12.3063 \\
-12.2766\end{array}$ & $\begin{array}{l}258 \\
256\end{array}$ \\
\hline in direct. & $\begin{array}{l}\text { Max sy } \\
\text { Min sy }\end{array}$ & $\begin{array}{l}{\left[\mathrm{N} / \mathrm{mm}^{\wedge} 2\right]:} \\
{\left[\mathrm{N} / \mathrm{mm}^{\wedge} 2\right]:}\end{array}$ & $\begin{array}{l}1.7852 \\
-1.9219\end{array}$ & $\begin{array}{l}257 \\
265\end{array}$ \\
\hline in direct. & $\begin{array}{l}\text { Max sz } \\
\text { Min sz }\end{array}$ & $\begin{array}{l}{\left[\mathrm{N} / \mathrm{mm}^{\wedge} 2\right]:} \\
{\left[\mathrm{N} / \mathrm{mm}^{\wedge} 2\right]:}\end{array}$ & $\begin{array}{l}2.1181 \\
-2.0302\end{array}$ & $\begin{array}{l}265 \\
257\end{array}$ \\
\hline Max Shear & $\begin{array}{l}\text { Max max } \\
\text { Min max }\end{array}$ & $\begin{array}{l}x T\left[\mathrm{~N} / \mathrm{mm}^{\wedge} 2\right]: \\
\text { XT [N/mm^2]: }\end{array}$ & $\begin{array}{l}9.5952 \\
0.0017\end{array}$ & $\begin{array}{l}258 \\
0\end{array}$ \\
\hline Shear Stress & $\begin{array}{l}\text { Max txy } \\
\text { Min txy }\end{array}$ & $\begin{array}{l}{\left[\mathrm{N} / \mathrm{mm}^{\wedge} 2\right]:} \\
{\left[\mathrm{N} / \mathrm{mm}^{\wedge} 2\right]:}\end{array}$ & $\begin{array}{l}0.3900 \\
-4.5503\end{array}$ & $\begin{array}{l}271 \\
258\end{array}$ \\
\hline Shear Stress & $\begin{array}{l}\text { Max tyz } \\
\text { Min tyz }\end{array}$ & $\begin{array}{c}{\left[\mathrm{N} / \mathrm{mm}^{\wedge} 2\right]:} \\
{\left[\mathrm{N} / \mathrm{mm}^{\wedge} 2\right]:}\end{array}$ & $\begin{array}{l}1.0407 \\
-1.0200\end{array}$ & $\begin{array}{l}256 \\
258\end{array}$ \\
\hline Shear Stress & $\begin{array}{l}\text { Max txz } \\
\text { Min txz }\end{array}$ & $\begin{array}{l}{\left[\mathrm{N} / \mathrm{mm}^{\wedge} 2\right]:} \\
{\left[\mathrm{N} / \mathrm{mm}^{\wedge} 2\right]:}\end{array}$ & $\begin{array}{l}1.5875 \\
-5.1043\end{array}$ & $\begin{array}{l}265 \\
256\end{array}$ \\
\hline Main Stress & $\begin{array}{r}\text { Max s1 } \\
\text { Min s1 }\end{array}$ & $\begin{array}{l}{\left[\mathrm{N} / \mathrm{mm}^{\wedge} 2\right]:} \\
{\left[\mathrm{N} / \mathrm{mm}^{\wedge} 2\right]:}\end{array}$ & $\begin{array}{l}13.9065 \\
-0.2111\end{array}$ & $\begin{array}{l}258 \\
271\end{array}$ \\
\hline Main Stress & $\begin{array}{r}\text { Max s2 } \\
\text { Min s2 }\end{array}$ & $\begin{array}{l}{\left[\mathrm{N} / \mathrm{mm}^{\wedge} 2\right]:} \\
{\left[\mathrm{N} / \mathrm{mm}^{\wedge} 2\right]:}\end{array}$ & $\begin{array}{l}5.1075 \\
-5.1705\end{array}$ & $\begin{array}{l}258 \\
256\end{array}$ \\
\hline Main Stress & $\begin{array}{r}\text { Max s3 } \\
\text { Min s3 }\end{array}$ & $\begin{array}{l}{\left[\mathrm{N} / \mathrm{mm}^{\wedge} 2\right]:} \\
{\left[\mathrm{N} / \mathrm{mm}^{\wedge} 2\right]:}\end{array}$ & $\begin{array}{l}0.2028 \\
-13.8173\end{array}$ & $\begin{array}{l}269 \\
256\end{array}$ \\
\hline
\end{tabular}

Figura 8. Interpretación de resultados 
Los resultados numéricos se obtienen haciendo click en «File», los cuales se graban en un archivo de texto. Asimismo, en la Figura 7 mediante el código de colores se muestra los esfuerzos entre 0,0029 y $19,19 \mathrm{~N} /$ $\mathrm{mm}^{2}$ en los que se encuentra sometida la viga.

Igualmente, se muestra en la Figura 8, el desplazamiento de la viga en los 3 planos principales $(X, Y, Z)$, resultando un valor máximo de $0,135 \mathrm{~mm}$.

\section{CONCLUSIONES}

El estudio muestra el análisis de la viga sometida a una fuerza de $2000 \mathrm{~N}$, que genera un desplazamiento espacial máximo de $0,135 \mathrm{~mm}$.

El uso de herramientas CAE, facilita el cálculo tedioso que antiguamente se realizaba de forma manual. Asimismo, permite variar las condiciones tales como carga, material etc. y se puede analizar en otras condiciones.

El Mechanical Desktop en su versión 6.0 incorpora herramientas más avanzadas tales como CAE, el cual permite una máxima capacidad de cálculo.

Finalmente, la utilización de este tipo de software en la enseñanza de los cursos de Ingeniería es necesaria porque las tendencias de vanguardia así lo requieren. La Facultad de Ingeniería Industrial de la Universidad Nacional Mayor de San Marcos no debe ni puede ser ajena a esta realidad.

\section{BIBLIOGRAFÍA}

1. Autodesk. (2004). Guía del Usuario Mechanical Desktop 6.0. Autodesk, Inc. USA.

2. Gould, Lawrence S. (1998). El Estudio de Fragmentos más pequeños por Elementos Finitos (FEA) conduce a la Optimización de Piezas. Febrero - Marzo. En: http://www.LSGould.com

3. Martínez S., Luis (2004). Apuntes del Curso de Dibujo Industrial. Facultad de Ingéniería Industrial, UNMSM. Lima, Perú. 